\title{
Editorial
}

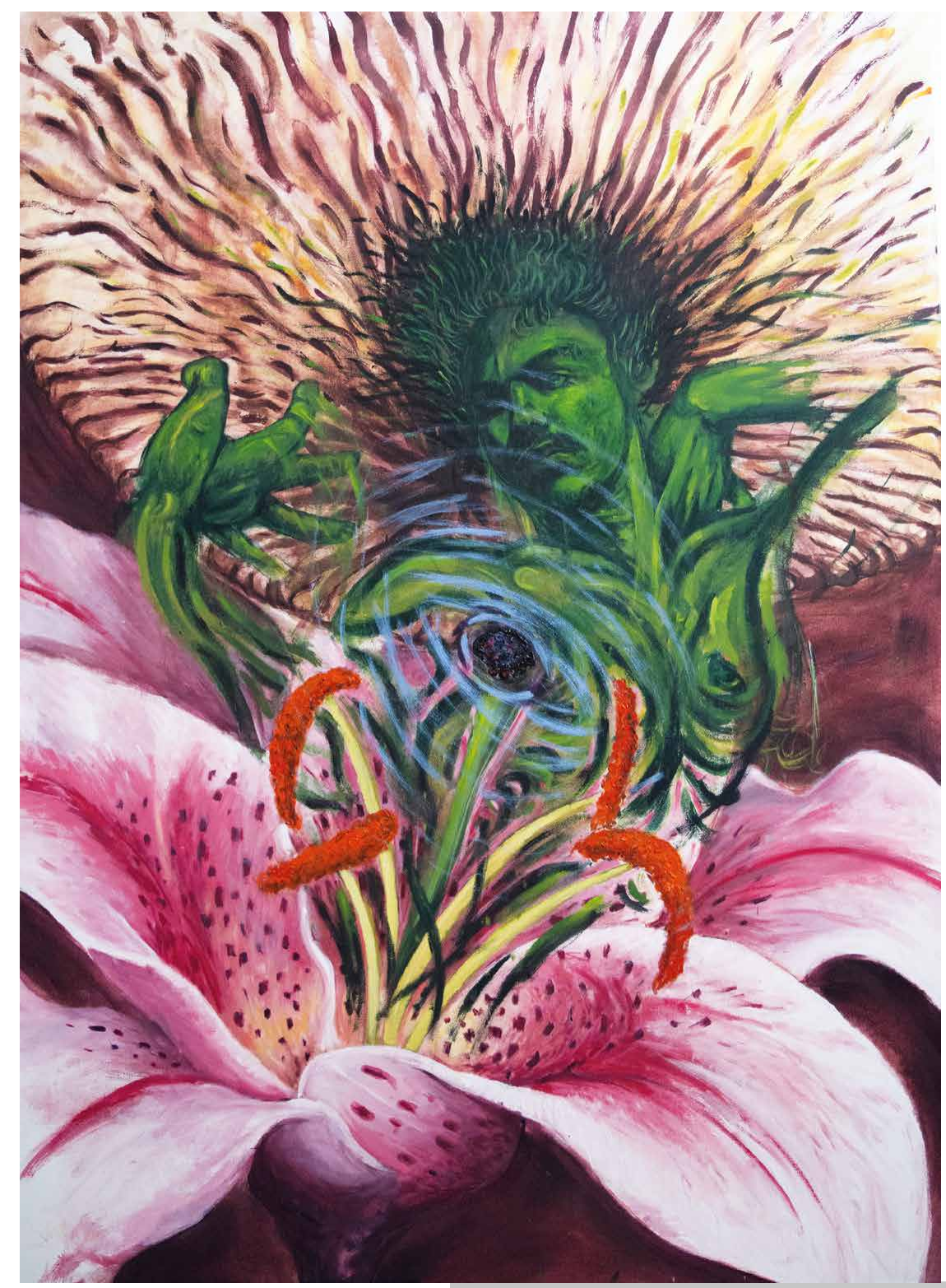

Artista invitado

Rafael Germán Rengifo Sánchez

Hacia donde voy, polen 1

De la serie El jardín detrás de mi cabeza Acrílico sobre lona

$105 \times 145 \mathrm{~cm}$

2020

Medellín 


\section{Los fondos para la reparación de las víctimas del conflicto armado en Colombia}

El punto cinco del Acuerdo Final, relativo al derecho de las víctimas a la verdad, la justicia, la reparación y la garantía de no repetición (Gobierno Nacional y FARC-EP, 2016), se ha convertido en uno de los temas más importantes en la fase del posconflicto con las Fuerzas Armadas Revolucionarias de Colombia-Ejército del Pueblo (FARC-EP). En este componente, los actores que antes se enfrentaban en la guerra se comprometieron con las víctimas a darles prioridad y mejorar sus condiciones de vida, como un derecho que tienen las víctimas a recibir de sus victimarios una compensación por los daños y pérdidas causados por el conflicto armado que protagonizaron.

A su vez, dentro de este gran tema relacionado con la justicia transicional, el aspecto que más polémicas ha causado es el de la reparación. Para unos, por ejemplo, la reparación debe ser «integral» (González, 2010; De Greiff, 2011; Zernova, 2007) y proponen el restablecimiento del statu quo de las víctimas, el cual consiste en dejarlas en una situación similar a la condición previa al momento en que ocurrieron los hechos de victimización, sobretodo, en asuntos de empleabilidad y propiedad. La propuesta es que en la restitución se «compense» $\mathrm{O}$ «indemnice» a la población victimizada con los bienes perdidos a raíz del conflicto.

Otros van más allá, proponen una reparación «transformativa» (Uprimny y Saffon, 2009). Además de exigir la restitución de derechos económicos, como ingresos y empleo, se enfocan en proponer mejoras en las condiciones de las víctimas, en términos sociales —educación, salud e inclusión-y personales -psicológicos-, independientemente de la situación inicial en que se encontraban (De Greiff, 2010; Uprimny y Saffon, 2009). De allí que incorporan en sus demandas tanto la dimensión económica - física o material- como la simbólica, política y social o colectiva, lo cual tiene que ver con el restablecimiento público de la dignidad de las víctimas y la garantía de no repetición. Es una propuesta que busca transformar a los sujetos y comunidades, que los lleve a una plena ciudadanía, con un acceso a todos sus derechos (Uprimny y Saffon, 2009). 
Las posturas sobre la reparación de las víctimas son variadas, enfatizan en lo económico, en lo psicológico y lo simbólico o cultural, proponen «una ayuda material, una asistencia psicológica y medidas simbólicas» (Reátegui, 2011, p. 53). Reparación necesaria, primero, por la necesidad que tienen las víctimas del conflicto de ser ayudadas, debido a su situación de pobreza, falta de educación y precarias condiciones de vida (Kiza y Rettberg, 2008, p. 71); segundo, por la urgencia que tienen las personas y comunidades de superar el trauma individual y social dejado por la guerra, y fortalecer la dignidad humana; $y$ tercero, debido al derecho que tienen las víctimas a que los victimarios se responsabilicen de sus acciones en medio de la guerra y que la justicia vaya más allá de una condena o sanción al victimario.

En todo caso, cualquiera sea el enfoque -económico o social, individual o colectivo, material o simbólico, integral o transformativo-, se requiere contar con recursos y organizaciones que atiendan el posconflicto y se ocupen de las labores que hagan posible la reparación (González, 2010, p. 631). En este texto nos ocuparemos de presentar y discutir uno de los temas que mayor controversia han generado en el posconflicto colombiano, se trata de los bienes que los actores desmovilizados han entregado al Estado para construir los fondos para financiar la reparación de las víctimas del conflicto armado.

\section{La evolución normativa de la reparación}

Colombia ha vivido un largo conflicto armado (Vargas, 2002), sin embargo, solo hasta la última década del siglo xx la legislación colombiana reconoció el derecho que tienen las víctimas a una reparación por los daños físicos, mentales, financieros y jurídicos causados por el conflicto. En los últimos veinticinco años el país ha evolucionado de forma constante en los derechos multidisciplinarios que tiene la población que ha sufrido los horrores de la guerra. Esta evolución normativa se puede apreciar a partir de cuatro hitos: el primero ocurrió en 1997 con la Ley 418; el segundo se dio con la Ley 975 de 2005 y, con ella, el Decreto 1290 de 2008; el tercero con la Ley 1448 de 2011; y finalmente, en 2018 con la Ley 1922.

A pesar de que Colombia tiene un largo conflicto armado y que cuenta con una significativa población afectada por este, solo a partir de la década de 1990 se consideró el derecho que tienen las víctimas a la reparación. Esta situación obedece a tres motivos: primero, durante este periodo se produjo la 
desmovilización de varias guerrillas y con ello la discusión sobre los deberes que tienen frente a los daños causados (Valencia, 2019); segundo, las reglas de juego cambian con el rediseño institucional que se creó con Constitución Política de 1991 y que entregó a la población nuevos derechos, entre ellos, la responsabilidad del Estado en la protección de los derechos fundamentales a las víctimas del conflicto, el cual puede exigirse a través de varios mecanismos, entre ellos, la acción de tutela; y tercero, los cambios de normas en el derecho internacional asociado a la guerra y con ella el acogimiento de Colombia a esta nueva normatividad internacional.

En este contexto de transformación normativa y de la realidad política es que surge el primer hito normativo de reconocimiento de las víctimas y su derecho a la reparación. Fue el gobierno de Ernesto Samper (1994-1998) el que adaptó la normatividad nacional en este nuevo escenario, atendiendo a la población afectada por el conflicto armado. Reconocimiento que quedó establecido con la Ley 418 de 1997, en la cual se elaboró un esquema de montos estandarizados para la reparación de las víctimas de delitos cometidos por grupos alzados en armas al margen de la ley — montos entregados de acuerdo a la tipología de las víctimas: desplazado, asesinato de un familiar, destrucción de la propiedad, entre otros-. En esta lógica, el Estado se comprometió a reparar económicamente a la población que ha sufrido las consecuencias nefastas de la guerra y a entregarle una indemnización monetaria a las víctimas.

Este modelo de monetización de la reparación fue revisado ocho años después, durante el doble gobierno de Álvaro Uribe (2002-2010) y en el entorno de las desmovilizaciones de las Autodefensas Unidas de Colombia (AUC) entre 2003 y 2006. La intensificación de la guerra durante este periodo provocó el aumento exponencial de las víctimas del conflicto (Grupo de Memoria Histórica, 2013) y debido a la presión de las organizaciones de víctimas, de la comunidad internacional y de las altas cortes se presentó un debate sobre el derecho de las víctimas a la verdad, la justicia y la reparación. La Ley 975 de 2005, conocida también como la Ley de Justicia y Paz, avanzó en este camino, pero debido a las dificultades en la materialización de la reparación y a las críticas de la Corte Constitucional se expidió el Decreto 1290 de 2008, con el que se construyó un modelo de reparación que superó la idea original de simple indemnización monetaria a una donde se incluyeron otros aspectos. 
No obstante, se detectaron vacíos en la normatividad, sobre todo en los temas de restitución de tierras, créditos para las víctimas y asistencia psicosocial, lo que provocó que en 2011, durante el doble mandato de Juan Manuel Santos (2010-2018), se trabajara en una nueva ley que corrigiera dichos vacíos de la normatividad existente. La Ley 1448 de 2011 o Ley de Víctimas y Restitución de Tierras —en adelante Ley de Víctimas- avanzó de manera significativa en la obtención del goce efectivo de los derechos fundamentales de las víctimas del conflicto armado, en «medidas judiciales, administrativas, sociales y económicas, individuales y colectivas para las víctimas de las infracciones al derecho Internacional Humanitario y normas internacionales de Derechos Humanos» (art. 1). Es una ley que incorporó temas claves para la reparación, como las medidas de no repetición, de satisfacción y de rehabilitación, así como programas de reparación completos y públicos dirigidos a la restitución e indemnización.

En cuanto a la indemnización, la ley mantiene la idea de una compensación en términos monetarios - monto de dinero-, a la cual tienen derecho las víctimas por haber sufrido ataques y afectaciones en su integridad y a los daños materiales — físicos — e inmateriales — mentalesprovocados por los crímenes (Unidad de Víctimas, s. f.); pero también amplía el derecho a la indemnización más allá del plano monetario y habla de la obligación que se tiene con las víctimas a atenderlas en el plano jurídico, médico, psicológico y social. En conclusión, la Ley de Víctimas se refiere a una reparación administrativa que, además de la indemnización monetaria, abarca otros aspectos psicosociales e individuales de las personas.

Finalmente, el último y más reciente hito de reparación está contenido en la Ley 1922 de 2018, con el que se reglamenta la Jurisdicción Especial para la Paz (JEP). Esta norma, además de establecer funciones para el nuevo organismo de justicia transicional, se ocupa de señalar mejores y mayores responsabilidades con las víctimas. Allí se habla de una reparación diferenciada que abarque todas las dimensiones de la afectación, incluyendo la simbólica; además, que avance en la transformación del entorno individual y social de la víctima. Responsabilidad ratificada en la Sentencia C-588 de 2019 de la Corte Constitucional: «las víctimas deben ser reparadas de manera adecuada, diferenciada, transformadora y efectiva por el daño que han sufrido. Establece entonces que la reparación comprende medidas de restitución, indemnización, rehabilitación, satisfacción y garantías de no repetición, en sus dimensiones individual, colectiva, material, moral y simbólica» (art 25). 
En conclusión, después de la elaboración de la Constitución Política de 1991el país ha dado significativos pasos en el reconocimiento que tienen las víctimas del conflicto armado a la reparación, a una compensación monetaria y unas obligaciones del Estado que debe abarcar «tanto generales como específicas, [...] garantizando el pleno y el libre ejercicio del derecho» (Rincón, 2010, p. 124). Es una responsabilidad del Estado — social de derecho- ofrecer un programa que permita una reparación plena de los derechos humanos, desde una visión integral y transformadora; y en este sentido buscar los recursos económicos y administrativos que hagan posible dicha reparación, entre ellos, los provenientes de la entrega de bienes por parte de los actores armados.

\section{Los bienes de los victimarios para reparación en Colombia}

Durante las primeras dos décadas del siglo XXI en Colombia se han presentado dos grandes desmovilizaciones de grupos al margen de la ley: entre 2003 y 2006, por parte de las AUC; y entre 2012 y 2016, por parte de las FARC-EP. La primera organización armada se caracterizó por tener una ideología y un accionar paraestatal y antisubversivo; la segunda, por el contrario, por ser una agrupación contraestatal y rebelde (Valencia, 2019). Debido a esta naturaleza contrapuesta, una vez en la Mesa de Conversaciones de La Habana, Cuba, la exguerrilla de las FARC-EP propuso un tratamiento diferencial en el tema de la justicia transicional. No querían ser tratados por el Estado colombiano de igual manera que lo hicieron con sus anteriores rivales militares. De allí que propusieron cambios sustanciales en el componente de justicia transicional, entre ellos, una variación en el organismo que se encargaría de recolectar los bienes para la reparación de las víctimas del conflicto armado.

De esta manera apareció en la institucionalidad dos mecanismos distintos para la administración de bienes para la reparación de víctimas en Colombia: por un lado, el Fondo para la Reparación de las Víctimas (FRV); y por el otro, la Sociedad de Activos Especiales (SAE). El primer fondo hace parte de la Unidad Administrativa Especial de Atención y Reparación Integral a las Víctimas o Unidad de Víctimas, la cual se encarga, en primer lugar, de administrar el Registro Único de Víctimas; en segundo lugar, de la administración de los recursos necesarios para la indemnización por vía administrativa en el marco de la Ley 975 de 2005 (arts. 166 al 168); 
y en tercer lugar, de la implementación de un Programa de Reparación Colectiva (arts. 151 y 152) (Corte Constitucional, Sentencia C-588, 2019).

La Unidad de Víctimas fue creada con el objetivo de garantizar a las víctimas su atención y debido cuidado, población que en la actualidad mayo de 2021—, según el Registro Único de Víctimas, suman 9113500 colombianos, una cifra enorme que representa aproximadamente 20\% de la población del país y que convierte a la labor de la Unidad en una tarea casi titánica. De allí que se entienda la lentitud con que vienen operando las indemnizaciones administrativas y judiciales -1 121176 víctimas por un monto de COL $\$ 7.8$ billones_ que se han realizado en una década de trabajo (Navarro, 2021, abril 9).

Para la atención de esta población, la Unidad de Víctimas cuenta, entre otras fuentes de financiamiento, con los recursos que los ex combatientes paramilitares y guerrilleros han entregado. La Ley 975 de 2005, intentando garantizar la reparación, el aporte de bienes y los recursos por parte de los desmovilizados para la indemnización, rehabilitación, satisfacción y garantías de no repetición, creó el FRV (art. 54). La Ley estableció que para el goce de los beneficios de la libertad los condenados deben aportar bienes al FRV. Los excombatientes beneficiados deben entregar al Estado los bienes obtenidos ilícitamente para la reparación de las víctimas. ${ }^{1}$

En esta lógica de trabajo, el FRV recibe, administra y monetiza «los bienes muebles e inmuebles y recursos que a cualquier título entreguen las personas o grupos armados ilegales; recursos provenientes del Presupuesto General de la Nación; donaciones en dinero o en especie; nacionales o extranjeras y por las nuevas fuentes de financiación» (art. 54); teniendo como mayores destinatarios a las víctimas de los grupos paramilitares, referidos en las sentencias de reparación integral de procesos que llevan los magistrados de Justicia y Paz.

El balance que se tiene del FRV, a febrero 10 de 2021, según el director de la Unidad de Víctimas, Ramón Rodríguez, es de 974 bienes rurales y 617 urbanos, distribuidos en 24 departamentos. Siendo Antioquia y Córdoba

\footnotetext{
${ }^{1}$ Debido a problemas en la implementación de la Ley de Justicia y Paz, en 2008, mediante el Decreto 1290, el Gobierno nacional creó el programa de reparación individual por vía administrativa, el cual tiene como objetivo reparar la violación de derechos por hechos cometidos por grupos armados organizados al margen de la Ley (GAOML) antes del 22 de abril de 2008, día de expedición del Decreto.
} 
los departamentos donde más se concentran estos bienes, 44,8\% y 14\%, respectivamente (Unidad de Víctimas, 2021, febrero 10). Fondo al que hoy todavía le siguen llegando bienes y recursos.

Por parte, las FARC-EP realizan la entrega de bienes a la SAE - no al Fondo de Reparación de la Unidad de Víctimas, como lo hacen los exparamilitares-. Esta decisión obedeció a la necesidad de la exguerrilla de separar su proceso de reparación del que vienen adelantando los antiguos grupos paramilitares. Así quedó consignado en el Decreto 1407 de 2017, donde se fijó la metodología de recepción: administración y cuidado de los bienes inventariados por las FARC-EP que van a un subfondo de la SAE.

Inicialmente, en agosto de 2017 la antigua guerrilla le entregó a la Misión de Verificación de la Organización de las Naciones Unidas y a la Fiscalía General de la Nación un inventario de bienes: 722 bienes inmuebles, COL $\$ 12000$ millones en efectivo, US $\$ 450$ 000, alrededor de 440000 gramos de oro, 24000 semovientes o cabezas de ganado, 50000 elementos que corresponden a muebles y enseres, además de vehículos y joyas (Redacción Colombia 2020, 2021, enero 6). Bienes tazados en alrededor de COL $\$ 1$ billón — cerca de medio billón monetizable-. A tres años de presentado el primer inventario se entregaron en su totalidad los USD \$450 000 y 252 500 gramos de oro, cifras que comparadas con la entregada por las AUC al FRV evidencia una gran distancia entre los aportes por las agrupaciones paramilitares y la exguerrilla. Por lo que se puede afirmar que las FARC-EP han sido la organización armada desmovilizada que más bienes ha aportado para la reparación de las víctimas en Colombia.

\section{La discusión: tensiones y retos}

Durante las últimas tres décadas la legislación colombiana ha evolucionado considerablemente en el reconocimiento del derecho de las víctimas a la verdad, la justicia, la reparación y la no repetición. Entre los temas de mayor polémica en la esfera pública ha sido el de la reparación. Aunque el Estado es el titular en la responsabilidad en la reparación con las víctimas, la legislación ha involucrado a los otros actores de la guerra como resultado de procesos de paz, así se puede evidenciar en los fondos creados para las AUC y las FARC-EP.

Aunque a simple vista esto sea lo correcto, pues esta división sirve para diferenciar las guerras en Colombia y sus víctimas, valorar de manera distinta 
las aportaciones que cada uno de los grupos de excombatientes realizó y fundamental para diferenciar escenarios de reconciliación, existen al menos dos tensiones que es necesario advertir:

La primera y más evidente tensión se da entre la postura que mantiene el Gobierno nacional y la de los exintegrantes de las organizaciones desarmadas, en el tema de los plazos para la entrega de los bienes para la reparación. En el caso de las AUC, el Gobierno nacional no impuso un cronograma de fechas o entregas de bienes. La adjudicación de sus bienes ha obedecido al resultado de labores de extinción de domino a cargo de la Fiscalía General de la Nación. Así, por ejemplo, en lo que va del 2021 el ente acusador le ha entregado al FRV 216 bienes, avaluados en COL\$167000 millones (Fiscalía General de la Nación, 2021, febrero 9).

Para las FARC-EP ocurrió algo similar, no se estableció ningún límite temporal para la entrega de los bienes, pero en este caso el gobierno de Iván Duque (2018-2022) sí ha presionado para la entrega de los aportes para la reparación. El presidente de la República ha acusado a esta organización y a sus líderes de incumplimiento en su compromiso de aportar para resarcir a las víctimas. Así lo dejó consignado en el Decreto 1080 de 2020, que obligaba a la exguerrilla a entregar la totalidad de los bienes inventariados para el 31 de diciembre de 2020. Según los dirigentes de la organización FARC, esta fue una decisión unilateral, no concertada con los máximos organismos encargados de la reincorporación - Consejo Nacional de Reincorporación (CNR) y Comisión de Seguimiento, Impulso y Verificación a la Implementación (CSIVI)—. En palabras de Pastor Alape — vocero en el CNR componente FARC—: «fue una decisión inconsulta del Gobierno para llevarnos al incumplimiento» (Comunes, 2020, diciembre 15).

A esta crítica que se le hace al Gobierno se le suman las otras que desde hace varios años vienen realizando sectores opositores y críticos del proceso, los cuales cuestionan la voluntad de la guerrilla en el objetivo de reparar a las víctimas y la entrega de la totalidad de sus bienes; en parte, basados en la expectativa que se tenía sobre la cantidad de bienes que esta exguerrilla poseía. Según Forbes Israel, las FARC-EP era la tercera agrupación terrorista más rica del mundo, con ingresos anuales superiores a los US $\$ 600$ millones (Portafolio, 2014, noviembre 12).

Todos estos elementos han puesto en entredicho los avances tempranos en materia de reparación a las víctimas por parte de los 
victimarios FARC-EP. Al respecto, la organización FARC ha manifestado que el retraso se presenta, en parte, por la reactivación del conflicto armado en zonas donde esta exguerrilla acumuló gran parte de sus bienes y ejercía control; ahora, algunas disidencias se han hecho con su control y otras han sido saqueados. Además, la informalidad e ilegalidad en la forma como fueron adquiridas estas propiedades ha provocado una complejización de la situación, la inseguridad que se vive en los exterritorios de dominio guerrillero pone en peligro a los firmantes de paz y con ello reducen las posibilidades de cuidado y mantenimiento de los bienes a aportar. $^{2}$

La organización FARC realizó, en el contexto de la guerra, una serie de inversiones sociales en infraestructura que deberían ser reconocidos como parte de sus aportes para la indemnización de las víctimas, por ejemplo, adecuación de carreteas — $3753 \mathrm{~km}$ de vías terciarias—, incluyendo $490 \mathrm{~km}$ que se encuentran dentro de áreas protegidas —en el Parque Nacional Serranía de Chiribiquete, la Reserva Nacional Natural Nukak y el Parque Nacional Natural Tinigua (Asociación Ambiente y Sociedad, 2019, junio 14)—. Pero estas inversiones no son reconocidas como aportes de los desmovilizados $y$, por el contrario, se les exige acelerar el proceso de entrega de bienes, sin contar con las garantías de seguridad o la asistencia técnica suficiente. En síntesis, el ahora partido Comunes siente que el gobierno Duque está ejerciendo una presión indebida y generando hostigamientos al proceso de reparación de víctimas.

La segunda tensión se presenta entre la gran cantidad de víctimas que esperan una reparación versus los escasos recursos que se tienen. Ante un universo de siete millones de víctimas que esperan ser reparadas en los próximos diez años, el Estado colombiano no tiene ni la capacidad ni los recursos para garantizar la reparación de esta población. Según informes de la Contraloría General de la República, la Ley de Víctimas se encuentra en saldo rojo y desfinanciada. Los cálculos que hace este organismo de control proyectan que hasta 2030 el Gobierno debe invertir COL\$357 billones para atender a los cerca de nueve millones de víctimas (El Tiempo, 2021, abril 9).

\footnotetext{
${ }^{2}$ El riesgo es muy alto y ante el precario desarrollo del Sistema Integral de Seguridad para el Ejercicio de la Política — contemplado en el punto dos del Acuerdo Final—y la estrategia de desmantelamiento de los grupos paramilitares (punto 3 ) reclamar estas propiedades o velar por su integridad mientras se resuelve su estatus legal representa un gran riesgo para los firmantes.
} 
Tan solo en las medidas de indemnización administrativa y colectiva se requieren de COL $\$ 48.7$ billones. Son cifras voluminosas, que equivalen a un poco más de $15 \%$ del Presupuesto General de la Nación —que para 2021 se fijó en COL\$313.9 billones-. Situación financiera que, sin duda, se verá aun más comprometida con las afectaciones económicas que esta produciendo la crisis económica y social de la pandemia, y que generará reajustes fiscales. De allí que se puede anticipar una situación aún mas crítica para el financiamiento de la atención y reparación de las víctimas del conflicto durante esta tercera década, por eso la necesidad de avanzar en la adecuada recepción, saneamiento e integración de los bienes entregados por los actores armados desmovilizados como medida de reparación a las víctimas.

La propuesta para el Estado es trabajar en la reducción de estas dos tensiones. El Gobierno, en lugar de aumentar polarizaciones en torno al cumplimiento de los grupos desmovilizados en temas de aportes a los fondos, debería estar interesado en fundamentar y materializar con más diligencia la reparación. Hay que recordar que tanto la legislación nacional como la internacional viene insistiendo en la obligación que tiene el Estado de realizar una reparación integral y transformadora de las víctimas del conflicto armado. En este sentido, algunas tareas que tiene el Gobierno son:

a. Trabajar en el involucramiento de las comunidades en las acciones reparadoras, en consultas más estrechas con los beneficiarios, sobre todo, en las reparaciones simbólicas y colectivas.

b. Buscar que el avance en las reparaciones sea coherente y complementario con los otros componentes de la justicia transicional: en el esclarecimiento de la verdad, la reconstrucción de la memoria histórica, la aplicación de la justicia y las reformas institucionales. De esta manera se le quitaría presión al tema monetario y financiero de la reparación de las víctimas.

c. Avanzar en un sistema balanceado de medidas de reparación individual y colectiva, materiales y simbólicas, para que las víctimas se sientan realmente reparadas. Una reparación integral y transformativa que contemple la restitución, la indemnización, la rehabilitación, la satisfacción y las garantías de no repetición. Una reparación adecuada a los daños cometidos, que se cumplan efectivamente en la realidad, que se ejecute en tiempos razonables, que tenga en cuenta las diferencias en los sujetos —edad, raza, estrato socioeconómico, entre otros-. 
d. Finalmente, tejer espacios que reparen su memoria e identidad. Debe mantenerse en el centro a las víctimas, evitando la polarización o las acusaciones mutuas de incumplimiento. A ellas se debe seguir rindiendo homenaje y por ellas no se debe recaer como sociedad en situaciones que persistan en el conflicto y generen mayor victimización. Ya la población civil ha sufrido lo suficiente con los múltiples conflictos armados.

\section{Germán Darío Valencia Agudelo (Colombia)* Fredy Alexánder Chaverra Colorado (Colombia)**}

\section{Referencias bibliográficas}

1. Asociación Ambiente y Sociedad. (2019, junio 14). Vías terciarias: otra forma de penetrar el corazón de la selva colombiana. https://www.ambienteysociedad.org. co/vias-terciarias-otra-forma-de-penetrar-el-corazon-de-la-selva-colombiana/

2. Colombia. Congreso de la República. Ley 418. (26 de diciembre de 1997). Por la cual se consagran unos instrumentos para la búsqueda de la convivencia, la eficacia de la justicia y se dictan otras disposiciones. https://www.unidadvictimas.gov.co/sites/ default/files/documentosbiblioteca/ley-418-de-1997.pdf

3. Colombia. Congreso de la República. Ley 975. (25 de julio de 2005). Por la cual se dictan disposiciones para la reincorporación de miembros de grupos armados organizados al margen de la ley, que contribuyan e manera efectiva a la consecución de la paz nacional y se dictan otras disposiciones para acuerdos humanitarios. https://www.unidadvictimas.gov.co/sites/default/files/ documentosbiblioteca/ley975de2005.pdf

4. Colombia. Congreso de la República. Ley 1448. (10 de junio de 2011). Por la cual se dictan medidas de atención, asistencia y reparación integral a las víctimas del conflicto armado interno y se dictan otras disposiciones. https://www.unidadvictimas. gov.co/sites/default/files/documentosbiblioteca/ley-1448-de-2011.pdf

5. Colombia. Congreso de la República. Ley 1922. (18 de julio de 2018). Por medio de la cual se adoptan unas reglas de procedimiento para la Jurisdicción

\footnotetext{
* Director revista Estudios Políticos. Economista. Especialista en Gerencia Social. Magíster en Ciencia Política. Doctor en Estudios Políticos. Grupo Hegemonía, guerras y conflictos, Instituto de Estudios Políticos, Universidad de Antioquia UdeA. Calle 70 No. 52-21, Medellín, Colombia. Correo electrónico: german.valencia@udea.edu.co - Orcid: 0000-0002-6412-6986 - Google Scholar https:// scholar.google.es/citations?user=7Sm8z3MAAAAJ\&hl=es

${ }^{* *}$ Politólogo. Grupo Hegemonía, guerras y conflictos, Instituto de Estudios Políticos, Universidad de Antioquia UdeA. Calle 70 No. 52-21, Medellín, Colombia. Correo electrónico: fredy.chavera@udea. edu.co
} 
Especial para la Paz. http://www.secretariasenado.gov.co/senado/basedoc/ ley_1922_2018.html

6. Colombia. Corte Constitucional. Sala Plena de la Corte Constitucional. Sentencia C-588. (5 de diciembre 2019). https://www.corteconstitucional.gov.co/ relatoria/2019/C-588-19.htm

7. Colombia. Departamento Administrativo de la Presidencia de la República. Decreto 1080. (31 de julio de 2020). Por medio del cual se modifica y adiciona el Decreto 1407 de 2017, modificado parcialmente por el Decreto 205 de 2020. https:// dapre.presidencia.gov.co/normativa/normativa/DECRETO $\% 201080 \% 20$ DEL $\% 20$ $31 \% 20$ DE $\% 20 J U L I O \% 20 D E \% 202020$.pdf

8. Colombia. Ministerio del Interior y de Justicia. Decreto 1290. (22 de abril de 2008). Por el cual se crea el programa de reparación individual por vía administrativa para las víctimas de los grupos armados organizados al margen de la ley. https://www.unidadvictimas.gov.co/sites/default/files/documentosbiblioteca/ decreto1290del22deabril2008.pdf

9. Comunes. (2020, diciembre 15). Estamos cumpliendo con la disposición de bienes para la reparación. https://partidofarc.com.co/farc/2020/12/15/estamoscumpliendo-con-la-disposicion-de-bienes-para-la-reparacion/

10. De Greiff, Pablo. (2010). Los esfuerzos de reparación en una perspectiva internacional: el aporte de la compensación al logro de la justicia imperfecta. Estudios Socio-Jurídicos, 7, pp. 153-199. https://revistas.urosario.edu.co/index.php/ sociojuridicos/article/view/468

11. De Greiff, Pablo. (2011). Justicia y reparaciones. En: Reátegui, Félix (ed.). Justicia transicional: manual para América Latina (pp. 407-440). Brasilia y Nueva York: Comisión de Amnistía, Ministerio de Justicia y Centro Internacional para la Justicia Transicional.

12. El Tiempo. (2021, abril 9). Los graves hallazgos de Contraloría con plata de reparación a víctimas. https://www.eltiempo.com/justicia/investigacion/victimashallazgos-de-la-contraloria-sobre-recursos-para-reparacion-579676

13. Fiscalía General de la Nación. (2021, febrero 9). Fiscalía entregará bienes que superan los $\$ 167.000$ millones para reparación a víctimas de las autodefensas. https://www.fiscalia.gov.co/colombia/justicia-transicional/fiscalia-entregara-bienesque-superan-los-167-000-millones-para-reparacion-a-victimas-de-las-autodefensas/

14. Gobierno Nacional y Fuerzas Armadas Revolucionarias de Colombia-Ejército del Pueblo (FARC-EP). (2016). Acuerdo Final para la Terminación del Conflicto y la Construcción de una Paz Estable y Duradera. Bogotá, D. C.: Imprenta Nacional.

15. González, Alexander. (2010). Justicia transicional y reparación a las víctimas en Colombia. Revista Mexicana de Sociología, 72 (4), pp. 629-658.

16. Grupo de Memoria Histórica. (2013). ¡Basta ya! Colombia: Memorias de guerra y dignidad. Bogotá, D. C.: Imprenta Nacional. 
17. Kiza, Ernesto y Rettberg, Angelika (comps.). (2008). Reparación en Colombia: ¿Qué quieren las víctimas? Retos, desafíos y alternativas para garantizar la integralidad. Bogotá, D. C.: Profis GTZ.

18. Navarro, Cristina. (2021, abril 9). Así avanza la reparación de más de 9 millones de víctimas colombianas. Caracol Radio. https://caracol.com.co/radio/2021/04/09/ nacional/1617977258_014321.html

19. Portafolio. (2014, noviembre 12). Las FARC son el tercer grupo terrorista más rico del mundo. https://www.portafolio.co/internacional/farc-son-tercer-grupoterrorista-rico-mundo-43474

20. Reátegui, Felix. (ed.) (2011). Justicia transicional: manual para América Latina. Brasilia y Nueva York: Comisión de Amnistía, Ministerio de Justicia y Centro Internacional para la Justicia Transicional.

21. Redacción Colombia 2020. (2021, enero 6). ABC de la entrega de bienes de las Farc: entienda la controversia entre gobierno y exguerrilla. El Espectador. https:// www.elespectador.com/colombia2020/pais/abc-de-la-entrega-de-bienes-de-farcentienda-la-pelea-entre-gobierno-y-exguerrilla/

22. Rincón, Tatiana. (2010). Verdad, justicia y reparación. La justicia de la justicia tradicional. Bogotá, D. C.: Universidad del Rosario.

23. Unidad para la Atención y Reparación Integral a las Victimas. (2021, febrero 10). La Unidad hace balance de los bienes administrados por el Fondo para la Reparación. https://www.unidadvictimas.gov.co/es/fondo-de-reparaciones/la-unidadhace-balance-de-los-bienes-administrados-por-el-fondo-para-la

24. Unidad para la Atención y Reparación Integral a las Victimas. (s. f.). Reparación integral individual. UARIV. https://www.unidadvictimas.gov.co/es/reparacionintegral-individual/286

25. Uprimny, Rodrigo y Saffon, María Paula. (2009). Reparaciones transformadoras, justicia distributiva y profundización democrática. En: Díaz, Catalina; Sánchez, Nelson; Uprimny, Rodrigo (eds.). Reparar en Colombia: los dilemas en contextos de conflicto, pobreza y exclusión (pp. 31-70). Bogotá, D. C.: Centro Internacional para la Justicia Transicional (ICTJ) y Centro de Estudios de Derecho, Justicia y Sociedad (DeJuSticia).

26. Valencia, Germán. (2019). Organizarse para negociar la paz. Gobernanza de la paz negociada en Colombia, 1981-2016. Medellín: Universidad de Antioquia.

27. Vargas, Alejo. (2002). Las fuerzas armadas en el conflicto colombiano antecedentes y perspectivas. Bogotá, D. C.: Intermedio.

28. Zernova, Margarita. (2007). Restorative Justice: Ideals and Realities. Hampshire: Ashgate. 